\title{
Negative afterimages and the McCollough effect
}

\author{
R. H. DAY and W. R. WEBSTER \\ Monash University, Clayton, Victoria, Australia
}

\begin{abstract}
Four experiments were conducted to test earlier claims about the relationship between the negative afterimage and the McCollough effect. The first claim (Hansel \& Mahmud, 1978) is that longlasting afterimages occur when induced by the same alternating-stimulus procedure as that used to induce the McCollough effect. The second claim (Murch \& Hirsch, 1972) is that afterimages can themselves induce McCollough effects if they are induced and paired sequentially with grating patterns. In testing these claims, a reliable computer-controlled color-cancellation technique developed earlier was used to measure the apparent color of both afterimages and McCollough effects objectively. No support was found for the first claim following alternative presentation of two homogeneously colored regions for total periods of $5 \mathrm{~min}$ (Experiment 1) and $20 \mathrm{~min}$ (Experiment 2). The second claim was fully supported: After an induction period of $7.3 \mathrm{~min}$, a McCollough effect occurred for a red-vertical pairing but not for a green-horizontal pairing (Experiment 3); but after an induction period of $20 \mathrm{~min}$, McCollough effects occurred strongly for both pairings (Experiment 4). The theoretical implications of these outcomes are considered in the context of recent theories of color and pattern processing in the visual system.
\end{abstract}

The McCollough effect (McCollough, 1965) usually follows alternating inspection of two stimulus patterns different in both color and orientation. For example, after inspection of gratings with red and black vertical bars and green and black horizontal bars presented repeatedly in alternation for about $10 \mathrm{sec}$ over about $10 \mathrm{~min}$, the same gratings in black and white appear colored. The white bars of the vertical grating appear pale green and those of the horizontal grating pink. Typically, the induced colors are nearly complementary to those of the inducing patterns; they depend on the orientation of the patterns (reversing when the head is tilted through about $90^{\circ}$ ); they do not require steady fixation of the patterns; and they are of relatively long duration, lasting in some cases for weeks (see Stromeyer, 1978). Since apparent color is dependent on the orientation of the inducing stimulus, the McCollough effect is included in the class referred to as contingent aftereffects. These are characterized by a contingency between an apparent property of the aftereffect, such as its color, and a particular property of the stimulus pattern, such as its orientation.

The negative color afterimage follows relatively brief fixation of a colored stimulus. When, after a minute or so, the gaze is shifted from the colored stimulus to an achromatic surface, the afterimage is visible as a pattern of the same shape in complementary colors. For example, if, after fixating a point on a circular pattern consisting of vertical red and green bars, the gaze is transferred to a gray surface, the afterimage appears as a circular pattern of pale green and pink bars. The effect is not orientation-contingent and generally requires steady fixation of the stimulus pattern. Depending on stimulus in-

Correspondence may be addressed to R. H. Day, Department of Psychology, Monash University, Clayton, Victoria 3168, Australia. tensity and duration, the afterimage typically lasts for a minute or so (Graham, 1965).

Because of the different conditions necessary to generate the McCollough effect and the negative afterimage, and because of the marked difference in their durations, it has been generally accepted that they are mediated by different processes (Harris \& Gibson, 1968). However, the distinction between the two effects was blurred by the outcomes of an experiment reported by Hansel and Mahmud (1978), in which afterimages were induced by a procedure resembling that used for induction of the McCollough effect. Hansel and Mahmud presented two rectangular "split-field" stimuli in alternation. In one stimulus, the left half was red and the right half white, and in the other, the left half was white and the right half green. After $3 \mathrm{~min}$ of 10 -sec alternations, the subjects reported negative afterimages of the colored regions, which lasted as long as $\mathbf{4 0} \mathrm{min}$. After $\mathbf{2 0} \mathrm{min}$ of these alternating presentations, 1 subject reported a negative afterimage after $8 \mathrm{~h}$ and another after $3 \mathrm{~h}$. Hansel and Mahmud (1978) also reported the induction of a McCollough effect and a negative afterimage on the same region of the retina. These were opposite in color and long in duration. The data consisted of qualitative verbal reports by the subjects.

Hansel and Mahmud's (1978) demonstration of longlasting negative afterimages and of a McCollough effect independent of an afterimage at the same retinal locus bears closely on an explanation of the latter. To account for the extended duration of the McCollough effect, a learning process has been proposed (Allan \& Siegel, 1986; Murch, 1976; Skowbo, 1986). The colored gratings are each conceived of as two separate stimulus components, a color and an achromatic grating. It is proposed that whên these two components are paired in the one pattern, color 
can act as an unconditioned stimulus and the grating as a conditioned stimulus. Thus the presentation of the achromatic grating after a number of paired color-grating presentations results in a conditioned response in the form of apparent color superimposed on the grating. To account for the complementary color of the conditioned response, it is assumed that the unconditioned stimulus evokes a negative afterimage. An explanation of the McCollough effect in terms of a conditioning-like process could not be valid if Hansel and Mahmud's (1978) finding that the McCollough effect and a negative afterimage occur independently at the same retinal locus is correct. To preserve the conditioning model, it would have to be assumed that two negative afterimages of opposed complementary colors develop simultaneously at the same retinal locus.

In another study, Murch and Hirsch (1972) showed that negative afterimages can themselves generate McCollough effects complementary in color to the afterimages. A homogeneous green stimulus followed by a vertical black and white grating was alternated with a homogeneous red stimulus followed by a horizontal black and white grating. Thirteen of 28 subjects reported a "negative" McCollough effect; vertical white bars appeared green and horizontal white bars appeared red. In addition, 7 subjects reported only half of the effect-that is, either the vertical bars appeared green or the horizontal bars appeared red. Eight subjects reported no effect. Murch and Hirsch explained this version of the McCollough effect in terms of the superimposition of the negative afterimages induced by the green and red stimuli onto the subsequently presented gratings. For example, after replacement of the green stimulus, a red afterimage was superimposed on the vertical achromatic grating. As a result, a red contingent aftereffect, the same color as the inducing stimulus, was generated. Murch and/Hirsch (1972) also found that this afterimage-produced McCollough effect was weakened by increasing the time interval between removal of the colored stimulus and its replacement by the grating. With intervals of $20-50 \mathrm{sec}$, most of the effect has dissipated. This implied that the afterimage faded after about 20-50 sec, so that it could no longer serve as an effective stimulus for generating the McCollough effect.

The results from the experiments by Hansel and Mahmud (1978) and Murch and Hirsch (1972) bear closely on the explanation of the McCollough effect. The former seriously question an explanation couched in terms of a conditioning process, and the latter indicate that a negative afterimage can serve as a substitute for a colored stimulus in the induction of the effect. For these reasons, confirmation using the quantitative color-cancellation procedure described earlier (Webster, Crassini, \& Willenberg, 1987; Webster, Day, \& Willenberg, 1988) was considered desirable. Four experiments are reported here. In Experiments 1 and 2, we were concerned with whether negative afterimages of long duration occur following induction by a procedure similar to that for induction of the McCollough effect. It should be pointed out straightaway that since no afterimages occurred even after $20 \mathrm{~min}$ of induction, the question of duration did not arise. Experiments 3 and 4 were designed to check Murch and Hirsch's (1972) finding that a McCollough-like effect occurs when a colored stimulus and an achromatic grating are sequentially paired and alternated with another pair of different color and orientation.

\section{EXPERIMENT 1}

The aim in Experiment 1 was to establish whether a negative afterimage occurs following an induction routine similar to that for the McCollough effect-that is, after repeated, alternating presentations of a colored and an achromatic stimulus. The procedure matched closely that described by Hansel and Mahmud (1978).

\section{Method}

Subjects. There were 12 undergraduate subjects who participated as part of a laboratory course. All had normal color vision according to the Ishihara test (Kanehara Shuppon Co., 1974).

Apparatus and Stimulus Patterns. All stimuli were presented on a Barco color monitor (Model GD 33), using a real-time image processing system (Image Technology Inc., Model AP-512) interfaced with a computer (DEC LSI-11/73). The computer also recorded and scored the subjects' buttonpress color-cancellation responses (see below).

The inducing stimuli consisted of two vertically divided rectangles, which subtended a visual angle of $11.90^{\circ}$ horizontally and $10.2^{\circ}$ vertically. A fixation point was located at the center of the vertical dividing edge. One stimulus was red in the left half of the rectangle, with a dominant wavelength of $620 \mathrm{~nm}$ and an average luminance of $12.65 \mathrm{~cd} / \mathrm{m}^{2}$, as measured by a Minolta chromometer (Model L100), and white in the right half, with an average luminance of $18.31 \mathrm{~cd} / \mathrm{m}^{2}$. The second stimulus was green in the left half (dominant wavelength $515 \mathrm{~nm}$, average luminance $18.31 \mathrm{~cd} / \mathrm{m}^{2}$ ) and white in the right half, with the same average luminance as the white half of the first stimulus. The test stimulus was a vertically divided rectangle the same size as the inducing stimuli, with a fixation point at the center of the dividing line. Both halves were white, with the same luminance as the white halves of the inducing stimuli.

Procedure. An appearance of red or green in the test stimuli before (pretest) or after (posttest) induction could be canceled by pressing a red or a green button repeatedly. If the half field looked red, the red button was pressed repeatedly until the redness was canceled to whiteness, after which the white button was pressed once. The same routine was followed if the half stimulus appeared green. If the half stimulus initially appeared white, the white button was pressed once; and if the point of apparent whiteness was overshot in the direction of the opposite color, the appropriate button was pressed to reverse the direction of cancellation. Each press of the red button produced a decrease in the amount of red by increasing the proportion of green, and vice versa. The changes in color with each buttonpress were linear in terms of CIE coordinates over a range of 40 presses. This method of measuring the change in color has already been described in detail by Webster, Day, and Willenberg (1988).

The scoring system developed earlier (Webster, Day, \& Willenberg, 1988) was used. When the test stimulus appeared green, and red was added by pressing the green button to produce apparent whiteness, the number of buttonpresses necessary to do so were treated as positive. When the test stimulus appeared green, and red was added by pressing the green button to cancel the color to apparent white, the number of buttonpresses to do so was treated as nega- 
tive. The final score was the difference between pretest and posttest, taking sign into account.

The subjects sat $1 \mathrm{~m}$ from the Barco monitor screen and viewed the stimulus patterns binocularly with heads upright. Each subject underwent a short period of instruction and practice, followed by a pretest, induction, and a posttest. During instruction and practice, the subjects were shown the screen and the cancellation buttons and given practice in canceling real colors. In the induction phase, the subjects fixated the points at the center of the inducing stimuli while they were presented for $5 \mathrm{sec}$ in alternation, 30 times each. Thus the total induction period was $5 \mathrm{~min}$. At the end of this period, there was a 1-min interval to allow for the dissipation of any shortterm afterimages. This interval was also included in Hansel and Mahmud's (1978) procedure, presumably also to allow time for the dissipation of short-term effects. In the pre- and posttests, the subjects canceled any apparent red or green in the left and right halves of the test stimulus.

\section{Results and Discussion}

The mean scores are shown in Figure 1. The directions of cancellation for the left and right half fields were in the expected direction-positive (red added) to cancel apparent green in the left half field, and negative (green added) to cancel apparent red in the right half field. However, it can be seen from Figure 1 that the mean amounts of added color to the left (L) and right (R) fields was slight, $.75(S D=1.22)$ on the left and -.67 $(S D=1.50)$ on the right. Neither mean was significant $\left[t_{\mathrm{L}}(11)=2.14\right.$, $\left.p>.05 ; t_{\mathrm{R}}(11)=1.54, p>.05\right]$.

Hansel and Mahmud (1978), following essentially the same procedure as that in this experiment for less time ( $3 \mathrm{~min}$ ), reported that negative color afterimages were present up to $\mathbf{4 0}$ min after the cessation of induction. In the experiment reported here, there was no evidence for afterimages $1 \mathrm{~min}$ after cessation of induction by the alternating stimuli. The matter was pursued further in Experiment 2.

\section{EXPERIMENT 2}

Hansel and Mahmud (1978) also reported that following an induction period of $20 \mathrm{~min}$ with the two alternating stimuli, negative afterimages were apparent on the test stimuli for very long periods-in the case of 2 subjects, for some hours. It was thought prudent, therefore, to check the results of Experiment 1 by prolonging the period of induction for $20 \mathrm{~min}$.

\section{Method}

Subjects. There were 4 undergraduate subjects whose color vision according to the Ishihara test was normal.

Apparatus, Stimuli, ahd Procedure. The apparatus, inducing and test stimuli, and procedure were the same as in Experiment 1 . The only difference was in the induction phase, which consisted of 6010 -sec presentations of each of the two inducing stimuli. Thus the induction phase took $20 \mathrm{~min}$.

\section{Results and Discussion}

The mean score for the left half field was $-.25(S D=$ $.50)$, and for the right half field it was $+.25(S D=.80)$. These means are shown for comparison with those for Experiment 1, in Figure 1. As can be seen, both were smaller than those for the first experiment, and they were

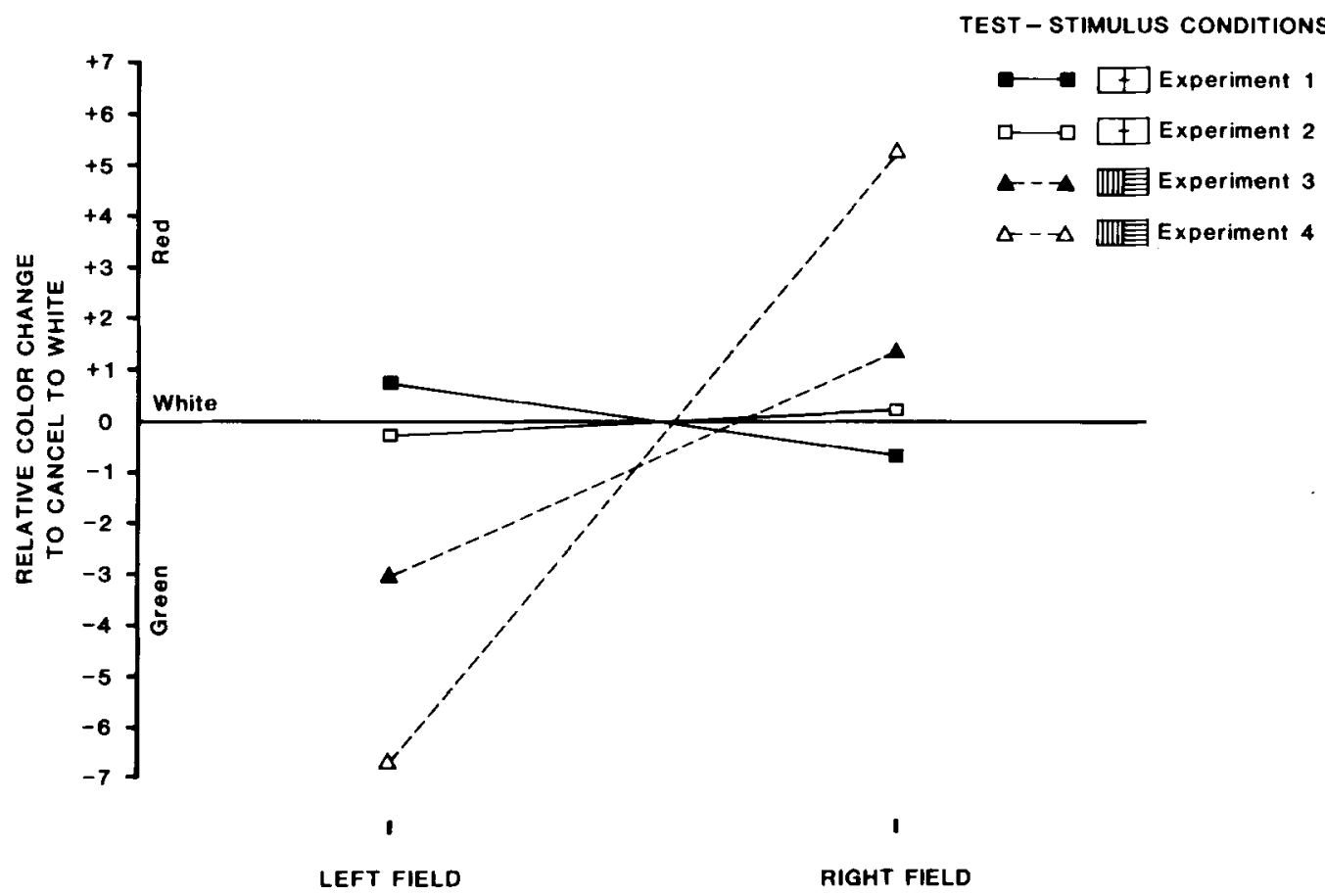

Figure 1. Mean color-cancellation scores for the four experiments. The score was the difference in color added to cancel apparent color between the pretest and posttest. Added green for cancellation was arbitrarily scored as negative and added red as positive. 
in the opposite direction-negative for the left half field and positive for the right half field. Neither mean was significantly different from zero $\left[t_{\mathrm{L}}(3)=1.00, p>.05\right.$; $\left.t_{\mathrm{R}}(3)=1.00, p>.05\right]$.

These results are entirely consistent with those of Experiment 1 , in showing that negative afterimages of color do not occur after protracted alternating stimulation by two spatially separated, colored stimuli. Thus, the claim that under this condition long-lasting afterimages occur (Hansel \& Mahmud, 1978) is not supported. It is not so much a question of whether negative afterimages generated by alternating presentation last for a long time as it is one of whether they occur at all. The quantitative data from Experiments 1 and 2 are unequivocal in showing that they do not survive for even a minute after cessation of stimulation. The consequences of this outcome for a theory of the McCollough effect are considered in the general discussion below.

\section{EXPERIMENT 3}

As pointed out above, Murch and Hirsch (1972) reported that a negative afterimage can be combined sequentially with an achromatic grating to produce a McCollough effect with a color complementary to the afterimage. The purpose in Experiment 3 was to check this finding by sequentially pairing one color with a vertical grating and another color with a horizontal grating and measuring any McCollough effects with the color-cancellation procedure described above.

\section{Method}

Subjects. Eight undergraduates, all with normal color vision according to the Ishihara test, participated.

Apparatus and Stimulus Patterns. The apparatus for generating and measuring the aftereffect was the same as that in Experiments 1 and 2. The pre- and posttest stimulus patterns consisted of a vertical and a horizontal black and white grating, which together formed a rectangular split field $11.9^{\circ}$ wide and $10.2^{\circ} \mathrm{high}$. The vertical grating formed the left half of the field, and the horizontal grating formed the right half. The spatial frequency of both gratings was $2 \mathrm{cpd}$, with a space-averaged luminance of $18.31 \mathrm{~cd} / \mathrm{m}^{2}$. There were two pairs of $11.9^{\circ} \times 10.2^{\circ}$ inducing stimuli. The first pair consisted of a red rectangle (dominant wavelength $620 \mathrm{~nm}$, luminance $13.32 \mathrm{~cd} / \mathrm{m}^{2}$ ) followed by a vertical achromatic grating ( $2 \mathrm{cpd}$, average luminance $18.31 \mathrm{~cd} / \mathrm{m}^{2}$ ). The second pair consisted of a green rectangle (dominant wavelength $515 \mathrm{~nm}$, luminance $\left.18.65 \mathrm{~cd} / \mathrm{m}^{2}\right)$ followed by a horizontal achromatic grating ( $2 \mathrm{cpd}$, luminance $18.31 \mathrm{~cd} / \mathrm{m}^{2}$ ).

Procedure. The subjects were seated $1 \mathrm{~m}$ from the monitor. Each session consisted of a pretest, a 5-min induction period, and a posttest. In the pre- and posttest, the subjects canceled any apparent red or green in the left (vertical) and right (horizontal) halves of the split-field stimulus, always in that order. During induction the red-vertical stimulus pair and the green-horizontal pair were presented in alternation. The duration of presentation for each pair was $10 \mathrm{sec}-5 \mathrm{sec}$ for the color and $5 \mathrm{sec}$ for the grating. Thus there were 15 presentations of the red-vertical pair and 15 of the greenhorizontal pair. It is emphasized that each component of the pair was presented in sequence- $5 \mathrm{sec}$ of color followed by $5 \mathrm{sec}$ of grating, with one pair following immediately on the other in an alternating sequence. There was again an interval of $1 \mathrm{~min}$ between the end of the induction period and the commencement of the pretest to allow time for any residual negative afterimage to dissipate.

The logic of the experiment was as follows: During induction, the red stimulus could be expected to generate a green afterimage on the vertical grating, and the green stimulus a red afterimage on the horizontal grating. Therefore, it was expected that in the posttest the vertical grating would appear red and require the addition of green to cancel it, whereas the horizontal grating would appear green and require the addition of red to cancel it. The scoring procedure was the same as in Experiment 1, with the addition of red treated as positive and that of green as negative.

\section{Results and Discussion}

The mean scores are plotted for comparison in Figure 1. The mean score of $-3.00(S D=3.55)$ following redvertical induction was in the expected direction (green added) and significant $[t(11)=2.40, p<.05]$. That of $1.38(S D=2.83)$ following green-horizontal induction was also in the expected direction (red added) but was not significant $[t(11)=1.40, p>.05]$. In brief, a significant McCollough effect resulted from sequentially pairing a red stimulus with a vertical grating, but not from sequentially pairing a green stimulus with a horizontal grating.

\section{EXPERIMENT 4}

In Murch and Hirsch's (1972) experiment, each colorgrating pair was presented in alternation for $20 \mathrm{sec}(10 \mathrm{sec}$ of color and $10 \mathrm{sec}$ of grating) for a total period of $7.3 \mathrm{~min}$. In Experiment 3 above, each pair was presented for $10 \mathrm{sec}(5 \mathrm{sec}$ of color and $5 \mathrm{sec}$ of grating) for $5 \mathrm{~min}$. It is conceivable that failure of the green-horizontal pair to produce a significant effect was due to insufficient induction. Therefore, in Experiment 4, both the duration and the frequency of presentation of the stimulus pairs were increased.

\section{Method}

Subjects. There were 9 undergraduate subjects, all with normal color vision.

Apparatus, Stimuli, and Procedure. The apparatus and stimulus patterns were the same as in Experiment 3. Except for the duration and frequency of presentation of the stimulus patterns, the procedure was also the same as in Experiment 3. The duration of each color-grating pair was increased from 10 to $20 \mathrm{sec}$ (10 sec of color and $10 \mathrm{sec}$ of grating), and each pair was presented 30 times in alternation with the other. Thus the total period of induction was $20 \mathrm{~min}$.

\section{Results and Discussion}

The mean scores are shown in Figure 1. It can be seen that both scores were substantially greater than those for the shorter induction periods in Experiment 3. The score for the red-vertical (RV) pair was $-6.70(S D=2.85)$, and that for the green-horizontal (GH) pair was 5.33 (SD $=5.94)$. Both scores were significant $\left[t_{\mathbf{R V}}(8)=8.17\right.$, $p<.01 ; t_{\mathrm{GH}}(8)=2.70, p<.05$ ]. It can also be noted that whereas an effect in the expected direction occurred for all 9 subjects with the red-vertical pair, it occurred 
for 6 of the 9 subjects for the green-horizontal pair. Thus, in terms of both the mean score and the number of subjects with scores in the expected direction, the effect for the red afterimage paired with a vertical grating was greater than that for a green afterimage paired with a horizontal grating.

\section{GENERAL DISCUSSION}

The results from Experiments 1 and 2 failed to confirm those reported by Hansel and Mahmud (1978). Not only were there no long-lasting afterimages following the alternating induction procedure, there were no measurable afterimages at all after $1 \mathrm{~min}$. In Hansel and Mahmud's (1978) experiment, all 6 subjects reported afterimages from red and green up to $40 \mathrm{~min}$ after induction. With yellow and blue inducing colors, negative afterimages were reported after $20 \mathrm{~min}$ by 3 subjects, after $10 \mathrm{~min}$ by 2 subjects, and after 5 min by 1 subject. The marked discrepancy between the results from Experiments 1 and 2 and those of Hansel and Mahmud are puzzling. It would be expected that the long-lasting effects reported by the latter would survive any minor variations in method between the two studies. However, it is to be noted that Hansel and Mahmud's subjects were required in the test period to report whether the stimulus was red, green, blue, yellow, neutral (white), or a combination of these colors. It is conceivable that the subjects had some awareness of the colors to be expected and responded accordingly. Apart from that possibility, no explanation of the discrepancy between the two studies can be given.

The results from Experiments 3 and 4 fully confirm those of Murch and Hirsch (1972), in showing that alternating sequential pairing of colors and gratings generates a form of the McCollough effect. The data confirm that the McCollough effects produced in this way are "negative," in that they are complementary in color to the negative afterimage rather than to the original stimulus colors. That is to say, it seems as if the color of the negative afterimage acts like a real color in conjunction with the grating pattern to generate a contingent color aftereffect.

One possible candidate to explain how the afterimage acts like a real color is the conditioning-like process (Allan \& Siegel, 1986; Murch, 1976; Skowbo, 1986) described briefly in the introduction. The essence of this explanation is that color can act as an unconditioned stimulus and the grating as a conditioned stimulus. To account for the complementary color of the McCollough effect-the conditioned response-it is assumed that the unconditioned stimulus evokes a negative afterimage process, which then gets elicited by the conditioned stimulus. However, even if this explanation is plausible for the McCollough effect generated by the conventional procedure involving colorgrating patterns, it is not so for the method adopted by Murch and Hirsch (1972) and in Experiments 3 and 4 above. With this procedure, the apparent color of the achromatic grating is complementary to that of the afterimage-it is the same as that of the original color. Thus it would be necessary to evoke some sort of second-order afterimage complementary to that of the first-order afterimage. It is not logically possible to have a second-order afterimage produced by any known mechanism capable of producing an afterimage. Virsu and Laurinen (1977) have shown the afterimages produced by stimuli of moderate intensity are a product of neural mechanisms in the retina. It is difficult to conceive how these retinal mechanisms could give rise to a second-order effect.

This difficulty for any explanation based on a conditioning process raises the question of whether the effect is due to adaptation processes in the visual system. Recent analysis of color processes at the retina (De Valois \& Jacobs, 1968; Gouras \& Zrenner, 1981) have shown that most cells carry information for both contrast and color. These cells also have concentric receptive fields, so they respond to all stimulus orientations. Thus, a homogeneous color stimulus should produce a negative afterimage in color cells that would also respond equally to both vertical and horizontal gratings. The selectivity of the McCollough effect, whether based on neural adaptation or learning processes, presumably takes place when the neural signal for an afterimage reaches an orientation detector in some cortical region, farther along from the cells with concentric receptive fields.

Gouras (1974) and Michael (1978) have proposed that double-opponent simple cells are the most likely candidates as the neural mechanisms mediating the McCollough effect. Michael (1978) has reported some preliminary evidence to show that simple cells respond in an appropriate way to an achromatic grating after adaptation to a colored grating. But these simple cells do not respond to homogeneous regions of color covering the receptive field of simple cells, since they have strong double-opponent interactions. Therefore, it seems unlikely that these cells would respond to a negative afterimage covering their receptive field. One possible mechanism is for the black and white test gratings to activate all the different types of retinal color cells encompassing the retinal area stimulated by the grating. A group of such cells would have previously been adapted by, for example, a red stimulus, and they would respond, say, to the white in the vertical grating as an afterimage. The effective stimulus for these cells would thus be a combination of the black and white vertical grating and a green afterimage. This output would then go to cortical detectors sensitive to green and vertical and thus give rise to the McCollough effect.

The data from these experiments bear on the model of color vision recently proposed by Livingstone and Hubel (1987). This model has been derived largely from cytochrome oxidase studies of monkey visual cortex. Livingstone and Hubel suggest that the cytochrome oxidase stains indicate high metabolic activity regions (or "blobs") in the visual cortex. Physiological studies show that they contain color-sensitive cells that are not sensitive to orientation. They also propose that there are three visual pathways going first to area 17 and then to area 18 . The first pathway is mainly a color system, which is not 
orientation sensitive. It involves parvocellular cells of LGN and the orientation-insensitive cells in area-17 blobs. The second pathway also involves parvocellular cells but interblob cells of area 17 as well. This pathway has high spatial acuity and orientation sensitivity, but most of the cells are not wavelength-sensitive. The third pathway implicates magnocellular cells. It has low spatial resolution and is probably insensitive to color. Livingstone and Hubel (1987) concede that the McCollough effect indicates that orientation and color selectivity must at some point converge, but they argue that the convergence must occur later than in areas 17 and 18 of the three systems. However, the afterimage data reported here suggest that the interaction between color and orientation occurs quite early in the system. There is other evidence to show that an afterimage and a real image interact. Georgeson and Turner (1985) and Burbeck and Kelly (1984) showed nearlinear additivity of contrast effects between afterimages of gratings and images of real gratings. In studies of spatial frequency filters, adaptation of gratings is used as a measure of filter bandwidth; it occurs in simple cells (Bishop, 1984), since they have the linearity required by spatial frequency theory. Thus the interaction between the afterimage and the real grating must take place either before or at the simple-cell level. If a similar process underlies the interaction of an afterimage and the McCollough effect, then the interaction of color and orientations would presumably take place much earlier than suggested by Livingstone and Hubel (1987).

\section{REFERENCES}

Allan, L. G., \& SIEgel, S. (1986). McCollough effects as conditioned response: Reply to Skowbo. Psychological Bulletin, 100, 388-393.

Bishop, P. O. (1984). The striate cortex: Feature detector or Fourier analyzer. Proceedings of the Auptralian Physiological \& Pharmacological Society, 15, 1-20.

Burbeck, C. A., \& Kelly, D. H. (1984). Role of local adaptation in the fading of stabilized images. Journal of the Optical Society of America (Series A), 1, 216-220.
De Valois, R. L., Jacobs, G. H. (1968). Primate color vision. Science, 162, 533-540.

Georgeson, M. A., \& TURner, R. S. E. (1985). Afterimages of sinusoidal, square-wave and compound gratings. Vision Research, 25, 1709-1720.

GouRAs, P. (1974). Opponent-colour cells in different layers of foveal striate cortex. Journal of Physiology, 238, 583-602.

Gouras, P., \& ZRENNER, E. (1981). Colour coding in primate retina. Vision Research, 21, 1591-1598.

GraHaM, C. H. (1965). Vision and visual perception. New York: Wiley.

Hansel, C. E. M., \& MAHMud, S. H. (1978). Comparable retention times for the negative colour afterimage and the McCollough effect. Vision Research, 18, 1601-1605.

Harris, E. S, \& Gibson, A. R. (1968). Is orientation-specific color adaptation vision due to edge detectors, afterimage, or "dipoles"? Science, 162, 1506-1507.

LivingStone, M. S., \& HuBEL, D. H. (1987). Psychophysical evidence for separate channels for the perception of form, color, and depth. Journal of Neuroscience, 7, 3416-3468.

McCollough, C. (1965). Color adaptation of edge-detectors in the human visual system. Science, 149, 1115-1116.

MiChaEL, C. R. (1978). Color vision mechanisms in monkey striate cortex: Simple cells with dual opponent-color receptive fields. Journal of Neurophysiology, 41, 1233-1249.

MURCH, G. M. (1976). Classical conditioning of the McCollough effect: Temporal parameters. Vision Research, 16, 615-619.

MurCH, G. M., \& Hirsch, J. (1972). The McCollough effect created by complementary afterimages. American Journal of Psychology, 85, 241-247.

Sxowвo, D. (1986). McCollough effects as conditioned responses? Reply to Allan and Siegel. Psychological Bulletin, 100, 394-397.

STROMEYER, C. F. (1978). Form-color aftereffects in human vision. In R. Held, H. W. Leibowitz, \& H.-L. Teuber (Eds.), Handbook of sensory physiology: Vol. VIII. Perception (pp. 97-142). Berlin: Springer.

VIRSU, V., \& LAURINEN, P. (1977). Long-lasting afterimages caused by neural adaptation. Vision Research, 17, 853-860.

Webster, W. R., Crassini, B., \& Willenberg, K. (1987). Simultaneous color contrast from McCollough effects is spatially contingent. Perception \& Psychophysics, 41, 402-408.

WeBSTER, W. R., DAY, R. H., \& WILlenBerG, K. (1988). Orientationcontingent color aftereffects are determined by real color, not induced color. Perception \& Psychophysics, 44, 43-49.

(Manuscript received July 11, 1988; revision accepted for publication May 8, 1989.) 